\title{
Analysis of Spaceborne Tandem Configurations for Complementing COSMO with SAR Interferometry
}

\author{
A. Moccia \\ Dipartimento di Scienza e Ingegneria dello Spazio "L.G. Napolitano," Università degli Studi di Napoli "Federico II," \\ Piazzale Tecchio 80, 80125 Napoli, Italy \\ Email: antonio.moccia@unina.it \\ G. Fasano \\ Dipartimento di Scienza e Ingegneria dello Spazio "L.G. Napolitano," Università degli Studi di Napoli "Federico II," \\ Piazzale Tecchio 80, 80125 Napoli, Italy \\ Email:g.fasano@unina.it
}

Received 29 June 2004; Revised 22 December 2004

\begin{abstract}
This paper analyses the possibility of using a fifth passive satellite for endowing the Italian COSMO-SkyMed constellation with cross- and along-track SAR interferometric capabilities, by using simultaneously flying and operating antennas. Fundamentals of developed models are described and potential space configurations are investigated, by considering both formations operating on the same orbital plane and on separated planes. The study is mainly aimed at describing achievable baselines and their time histories along the selected orbits. The effects of tuning orbital parameters, such as eccentricity or ascending node phasing, are pointed out, and simulation results show the most favorable tandem configurations in terms of achieved baseline components, percentage of the orbit adequate for interferometry, and covered latitude intervals.
\end{abstract}

Keywords and phrases: spaceborne SAR interferometry, multiplatform interferometry, cross-track interferometry, along-track interferometry, mission analysis.

\section{INTRODUCTION}

COSMO-SkyMed is the Italian constellation for high spatial and temporal resolution SAR imaging of the Earth $[1,2]$. COSMO stands for COnstellation of small Satellites for Mediterranean basin Observation and, basically, it consists of four satellites in sun-synchronous orbit, orbiting in the same plane and phased at $90^{\circ}$, each equipped with an advanced $X$ band SAR (synthetic aperture radar). Constellation orbital parameters are reported in Table 1.

The program has been approved and founded, the development is carried out by Alenia Spazio as prime contractor, under management of the Italian Space Agency (ASI), and the launch of the first satellite is scheduled in 2006.

The possibility of flying a passive satellite, that is equipped with a receiving-only antenna, in formation with COSMO-SkyMed for bistatic applications has been investigated in $[3,4]$. The study has been conducted assuming that no modifications should be included in design and opera-

This is an open access article distributed under the Creative Commons Attribution License, which permits unrestricted use, distribution, and reproduction in any medium, provided the original work is properly cited. tion of the main mission, in order to avoid both expensive redesign and checkout phases at this stage of COSMO development, and degradations of its nominal performance. This fifth satellite, named BISSAT (BIstatic Sar SATellite), could fulfill also interferometric applications, by selecting adequate tandem orbits, thus obtaining interferometric pairs without time decorrelation [5].

This paper analyses various tandem configurations of the proposed COSMO-BISSAT formation, aimed at cross-track (XTI) and along-track (ATI) interferometry. Several possible orbits have been considered for BISSAT, evaluating their characteristics versus potential interferometric applications.

Several authors have carried out performance evaluations of multistatic space configurations and have compared solutions presented in the literature mainly in terms of range and azimuth ambiguities and geometric decorrelation $[6,7]$, whereas this paper is aimed at describing main aspects and results of analytical models developed to compute the achievable interferometric baselines, their time histories along the selected orbits and relevant ground coverages. In particular, XTI baselines are investigated accounting for system capability to attain satisfactory phase errors and, hence, height measurement accuracy. Concerning ATI, a baseline study is 
TABLE 1: COSMO orbital parameters.

\begin{tabular}{lc}
\hline Semimajor axis $(a)$ & $6997.940 \mathrm{~km}$ \\
Eccentricity $(e)$ & 0.00118 \\
Argument of perigee $(\omega)$ & $90^{\circ}$ \\
Inclination $(i)$ & $97.87^{\circ}$
\end{tabular}

carried out to investigate radial velocity measurement capabilities.

The first part of the paper is devoted to the analysis of the critical baselines for SAR interferometry since they constitute the basic requirements to be fulfilled by the formation. Then, the model developed for propagating baseline components along the orbit is described in more detail. Finally, the proposed tandem configurations are introduced and simulation results indicate achievable performance in terms of baseline components, percentage of the orbit adequate for interferometry, covered latitude intervals. For the sake of presentation clarity, considered orbits have been divided in two groups: the coplanar tandem configurations, which apply when COSMO and BISSAT orbital planes are coincident and include the well-known cartwheel $[8,9]$, and pendulum tandem configurations $[10,11,12]$, which refer to noncoincident orbital planes.

\section{CRITICAL BASELINES FOR SAR INTERFEROMETRY}

Some values of baseline components, which are critical for interferometric processing, exist both in along- and crosstrack direction. A thorough analysis of these aspects can be found in $[13,14]$.

Objective of this paragraph is to review and apply these models to the system under study in order to define the baseline intervals which tandem on-orbit configurations must attain.

Let $x y z$ be a right-handed, Cartesian orbiting reference frame (ORF) whose origin coincides with COSMO position, with $z$-axis towards the geometrical center of the Earth, and $y$-axis opposite to the angular momentum vector, as shown in Figure 1. The interferometric baseline components, $B_{x}, B_{y}$, and $B_{z}$, coincide with BISSAT's coordinates in the ORF: $B_{x}$ is the along-track baseline, $B_{y}$ and $B_{z}$ are the horizontal and vertical components of the cross-track baseline.

As for cross-track interferometry is concerned, the minimum baseline condition has been calculated by imposing that the interferometric phase variation $d \Phi_{12}$ between adjacent targets is equal to the expected interferometric phase uncertainty $\sigma_{\Phi}$. As shown in $[15,16,17,18]$, it is possible to set up a phase error model that accounts for baseline separation, in particular; interferometric phase uncertainty decreases with baseline, due to an increasing capability in angular separation measurement. For the sake of consistency, it has been assumed a theoretical limit in baseline reduction: when the unavoidable phase noise consequent to signal-tonoise ratio $\left(\sigma_{\Phi}\right)$ is equal to the minimum phase measurement requirement, that is, the capability to detect the phase difference existing between adjacent targets at the same height.

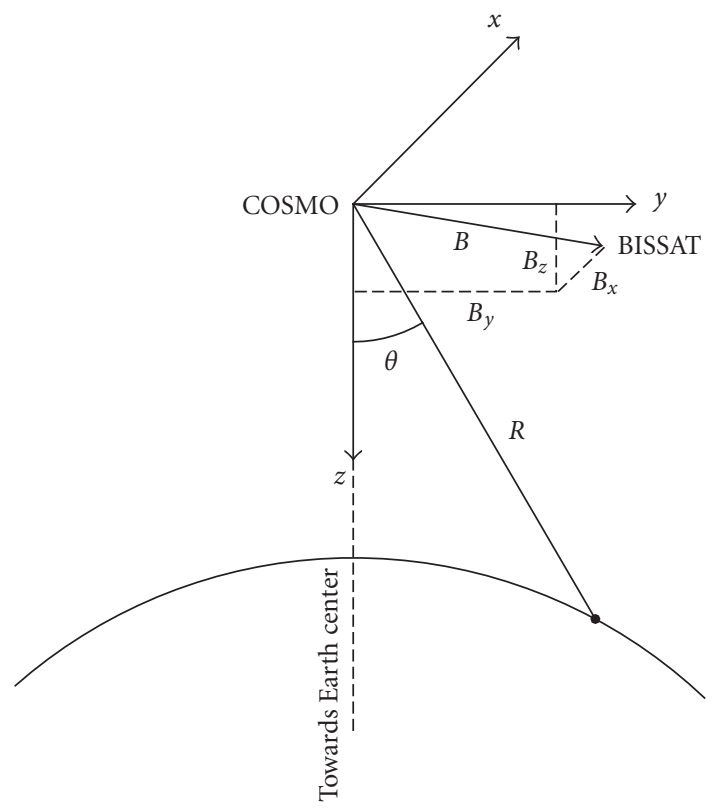

Figure 1: Orbiting reference frame and interferometric baseline.

The interferometric phase can be expressed as

$$
\Phi_{12} \cong \frac{2 \pi}{\lambda}\left(B_{z} \cos \theta-B_{y} \sin \theta\right),
$$

where $\theta$ represents the look angle.

Thus, the above phase variation can be computed as

$$
d \Phi_{12} \cong \frac{\partial \Phi_{12}}{\partial \theta} d \theta=\frac{2 \pi}{\lambda}\left(-B_{z} \sin \theta-B_{y} \cos \theta\right) d \theta .
$$

Since

$$
d \theta \cong \frac{d R_{g} \cos \theta}{R}
$$

where $d R_{g}$ is the ground-range resolution and $R$ is the slant range, the condition to be satisfied is

$$
\left|B_{z} \sin \theta+B_{y} \cos \theta\right| \geq \frac{\lambda R}{2 \pi d R_{g} \cos \theta} \sigma_{\Phi} .
$$

To express interferometric phase uncertainty $\left(\sigma_{\Phi}\right)$ as a function of signal-to-noise ratio (SNR), it has been assumed [5]

$$
\gamma_{0}=\frac{\mathrm{SNR}}{1+\mathrm{SNR}}
$$

and the phase standard deviation as a function of coherence $\left(\gamma_{0}\right)$ has been numerically computed, on the basis of the statistical distributions reported in [19].

Assuming $\theta=33.5^{\circ}$, slant-range resolution equal to $5 \mathrm{~m}$, SNR $=15 \mathrm{~dB}$, and four-look processing, a value of $53.10 \mathrm{~m}$ is obtained for the right-hand member in (4). In the case of coplanar orbits, $B_{y}=0$ and (4) becomes $B_{z} \geq 96.21 \mathrm{~m}$. 
The maximum-baseline configuration is determined by the phenomenon of baseline decorrelation, that is, the drop or even loss of the interferometric pair correlation because of excessively large antenna separation $[5,11,14,15]$. Although larger baselines allow a larger height measurement sensitivity, decorrelation determines a larger phase measurement noise. It can be mitigated by making use of multilook processing and an optimal baseline can be identified, accounting for shorter baselines problems too. However, in the following only the theoretical limit on maximum-baseline consequent to decorrelation will be accounted for.

From [5], in a single-pass case like that of COSMOBISSAT tandem, the following expression for the spatial correlation coefficient $(\rho)$ can be taken:

$$
\rho=1-\frac{\cos \Theta|\delta \theta| d R_{g}}{\lambda},
$$

where $\delta \theta$ is the difference in look angle for the two antennas and $\Theta$ is the local incidence angle, in the case of the aboveassumed COSMO geometry and flat terrain $\Theta=37.3^{\circ}$.

Introducing the so-called "effective baseline" $B_{\perp}$, that is, the baseline component normal to the direction of incidence [20], it results in

$$
|\delta \theta|=\frac{B_{\perp}}{R}
$$

and so, considering a drop to 0.5 of the spatial correlation coefficient, making substitutions, we obtain

$$
B_{\perp \max }=\frac{\lambda R}{2 \cos \Theta d R_{g}} .
$$

In particular, for the above system characteristics, in the case of coplanar orbits

$$
B_{z \max }=\frac{\lambda R}{2 d R_{g} \sin \theta \cos \Theta}=2.98 \mathrm{~km}
$$

while for pendulum configurations (cross-track baseline formed almost completely in horizontal direction),

$$
B_{y \max }=\frac{\lambda R}{2 d R_{g} \cos \theta \cos \Theta}=1.97 \mathrm{~km} .
$$

Regarding along-track interferometry, a range of $[75 \mathrm{~m}$, $150 \mathrm{~m}$ ] for $B_{x}$ will be derived in the following, under the assumption of performing oceanographic applications. Furthermore, since when only one of the two antennas is a transmitting/receiving one, the effective along-track baseline is half the along-track physical separation between the antennas, the time lag between the antennas must be in the range of about $[5,10]$ milliseconds.

In more detail, the upper limit of $B_{x}$ depends on the decorrelation of ocean echoes [20] and is obtained from

$$
\Gamma(t)=\gamma_{0} \exp \left(-\frac{t}{\tau_{s}}\right),
$$

where $\Gamma(t)$ is the instantaneous interferometric data coherence and $\gamma_{0}$ represents coherence for zero time lag (5), assuming that ocean decorrelation time $\tau_{s}$ is equal to 15 milliseconds, and accepting a coherence drop to 0.5. Of course, larger baselines could be adopted in other applications, when decorrelation is a less stringent constraint.

The along-track baseline lower limit, instead, is related to the achievable velocity measurement accuracy, and, above all, to collision risk avoidance. In the considered case, the maximum radial velocity $\left(V_{r \max }\right)$ that can be measured avoiding the necessity of phase unwrapping is in the range $[0.78,1.56]$ $\mathrm{m} / \mathrm{s}$, and the theoretical limit of measurement accuracy, that is [21],

$$
\frac{V_{r \max }}{\pi} \sigma_{\phi}
$$

is about $0.2 \mathrm{~m} / \mathrm{s}$ adopting four looks. However, it can be greatly improved if processing is based on more looks, although causing a reduced geometric resolution [20].

\section{INTERFEROMETRIC BASELINES EVALUATION}

In this section, the procedure for computing the interferometric baselines for any choice of the tandem orbital configuration will be pointed out. The inertial position of both satellites is known if the instantaneous value of six orbital parameters (right ascension of the ascending node $\Omega$, inclination $i$, semilatus rectum $p$, eccentricity $e$, argument of perigee $\omega$, and true anomaly $\nu$ ) are known. In the following the subscripts $C$ and $B$ will refer to COSMO and BISSAT. In practice, neglecting all perturbations and assigned the initial conditions, the true anomaly is the only parameter which varies with time, according to Kepler's equation [22]. Knowing COSMO's and BISSAT's orbital parameters at a given instant, it is possible to define two useful reference frames. Let $X_{C} Y_{C} Z_{C}$ be a geocentric, right-handed reference frame with $X_{C}$-axis directed towards COSMO ascending node and $Y_{C}$-axis opposite to its angular momentum vector, so that $X_{C} Z_{C}$ plane is coincident with COSMO orbital plane, while $X_{B} Y_{B} Z_{B}$ is the same frame based on BISSAT position (Figure 2); $x y z$ is the orbiting reference frame previously introduced (Figure 3). Obviously, in the case of coplanar configurations, $X_{B} Y_{B} Z_{B}$ and $X_{C} Y_{C} Z_{C}$ coincide.

BISSAT position in $X_{B} Y_{B} Z_{B}$ is given by

$$
\left[\begin{array}{c}
X_{B} \\
Y_{B} \\
Z_{B}
\end{array}\right]=r_{B} \cdot\left[\begin{array}{c}
\cos \left(\omega_{B}+\nu_{B}\right) \\
0 \\
\sin \left(\omega_{B}+\nu_{B}\right)
\end{array}\right],
$$

where

$$
r_{B}=\frac{p_{B}}{1+e_{B} \cos \nu_{B}} .
$$

The transformation matrix from $X_{B} Y_{B} Z_{B}$ to $X_{C} Y_{C} Z_{C}$ can be derived considering that the latter is obtained from the former by applying the following sequence of Euler angles $90^{\circ}-i_{B}, \Omega_{C}-\Omega_{B}, i_{C}-90^{\circ}$, with $90^{\circ}-i_{B}$ and $i_{C}-90^{\circ}$ around 


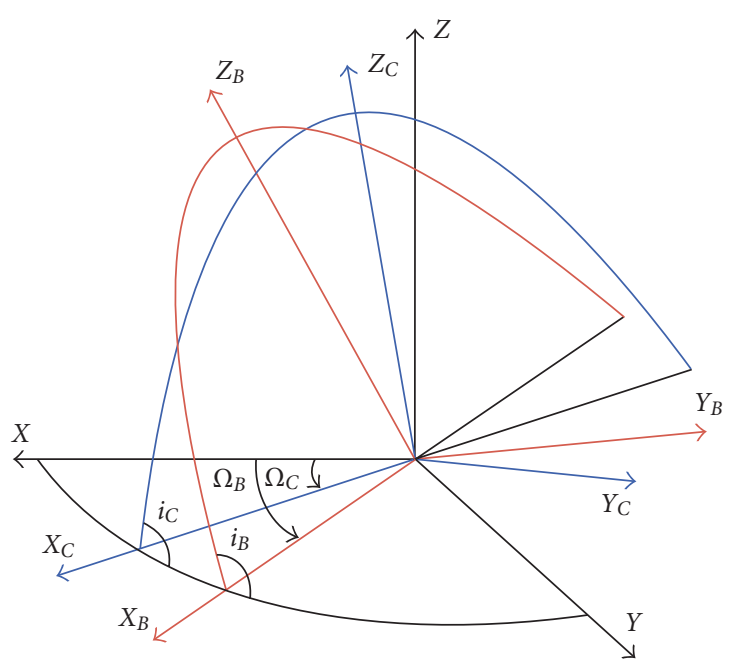

Figure 2: Geocentric reference frames.

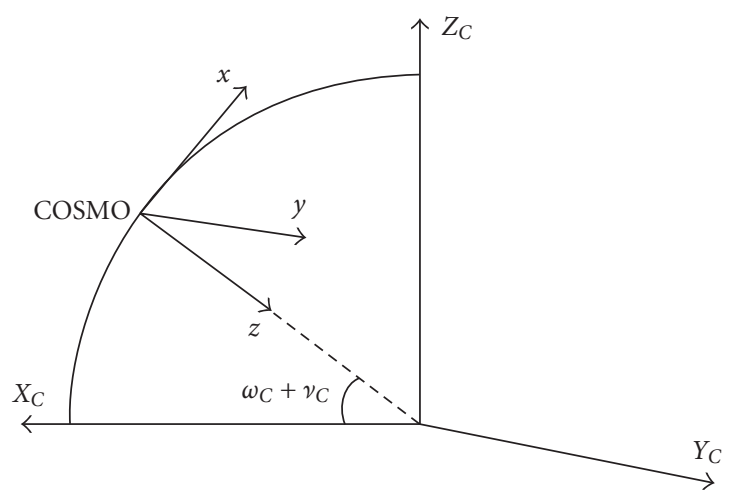

Figure 3: ORF and $X_{C} Y_{C} Z_{C}$.

the first axis and $\Omega_{C}-\Omega_{B}$ around the third axis, thus achieving

$M_{B \rightarrow C}=$

$\left[\begin{array}{cll}\cos \Delta \Omega & -\sin i_{B} \sin \Delta \Omega & -\cos i_{B} \sin \Delta \Omega \\ \sin i_{C} \sin \Delta \Omega & \sin i_{C} \sin i_{B} \cos \Delta \Omega+ & \sin i_{C} \cos i_{B} \cos \Delta \Omega+ \\ & +\cos i_{C} \cos i_{B} & -\cos i_{C} \sin i_{B} \\ \cos i_{C} \sin \Delta \Omega & \cos i_{C} \sin i_{B} \cos \Delta \Omega+ & \cos i_{C} \cos i_{B} \cos \Delta \Omega+ \\ & -\sin i_{C} \cos i_{B} & +\sin i_{C} \sin i_{B}\end{array}\right]$,

where $\Delta \Omega=\Omega_{B}-\Omega_{C}$.

Then, the passage from $X_{C} Y_{C} Z_{C}$ to $x y z$ is given by

$$
\left[\begin{array}{c}
x \\
y \\
z
\end{array}\right]=\left[\begin{array}{c}
0 \\
0 \\
r_{C}
\end{array}\right]+M_{o}\left[\begin{array}{c}
X_{C} \\
Y_{C} \\
Z_{C}
\end{array}\right]
$$

with

$$
M_{o}=\left[\begin{array}{ccc}
-\sin \left(\omega_{C}+\nu_{C}\right) & 0 & \cos \left(\omega_{C}+\nu_{C}\right) \\
0 & 1 & 0 \\
-\cos \left(\omega_{C}+\nu_{C}\right) & 0 & -\sin \left(\omega_{C}+\nu_{C}\right)
\end{array}\right] .
$$

This procedure allows propagation of the interferometric baselines for any initial condition. In particular, inclusion of orbital perturbations in propagating orbital parameters does not require any modification of the procedure for baselines evaluation. Furthermore, it is worth noting that COSMOBISSAT relative position within a single orbit can be described assuming unperturbed motion, while orbital perturbations help to foresee the long period evolution of the considered formation.

\section{TANDEM FLIGHT IN COPLANAR CONFIGURATIONS}

To describe the kinematics of coplanar configurations, it is useful to consider the relative motion of a satellite moving on an orbit of given eccentricity and semimajor axis, with respect to a reference point describing a reference trajectory. The selected reference trajectory is a Keplerian circular orbit lying in the satellite orbital plane, sharing the same mean motion $(n)$ of satellite elliptical orbit, hence the two orbits exhibit the same semimajor axes (i.e., the semimajor axis of the elliptical orbit is equal to the circular orbit radius) and orbital periods $(T)$.

Now, let $x_{o} z_{o}$ be an orbiting reference frame $\left(\mathrm{ORF}_{O}\right.$, in the following the subscript $O$ will refer to the reference orbit) whose origin coincides with the position of the reference point ( $x_{0}$-axis directed as the velocity vector, $z_{0}$-axis in nadir direction); assuming that mean anomaly $M$ initial value is

$$
M \equiv-\omega+\theta_{0},
$$

where $\theta_{0}$ is the initial value of the true anomaly of the reference point with respect to the ascending node, the following equations can be derived by a series expansion in powers of eccentricity [23]:

$$
\begin{aligned}
x_{o}(t)= & 2 a e \sin \left(n t+\theta_{0}-\omega\right)+\frac{a e^{2}}{4} \sin 2\left(n t+\theta_{0}-\omega\right) \\
& +\frac{a e^{3}}{24}\left[7 \sin 3\left(n t+\theta_{0}-\omega\right)-9 \sin \left(n t+\theta_{0}-\omega\right)\right] \\
& +O\left(e^{4}\right), \\
z_{o}(t)= & a e \cos \left(n t+\theta_{0}-\omega\right)+\frac{a e^{2}}{2}\left[1-\cos 2\left(n t+\theta_{0}-\omega\right)\right] \\
& +\frac{3}{8} a e^{3}\left[\cos \left(n t+\theta_{0}-\omega\right)-\cos 3\left(n t+\theta_{0}-\omega\right)\right] \\
& +O\left(e^{4}\right),
\end{aligned}
$$

where $t$ is the time elapsed since initial instant. By truncating the series at first order in eccentricity, the satellite trajectory with respect to the reference point is an ellipse whose center coincides with the reference point and with principal axes directions coincident with $x_{o} z_{o}$ directions. In particular, horizontal and vertical semiaxes have length $2 a e$ and $a e$, respec- 


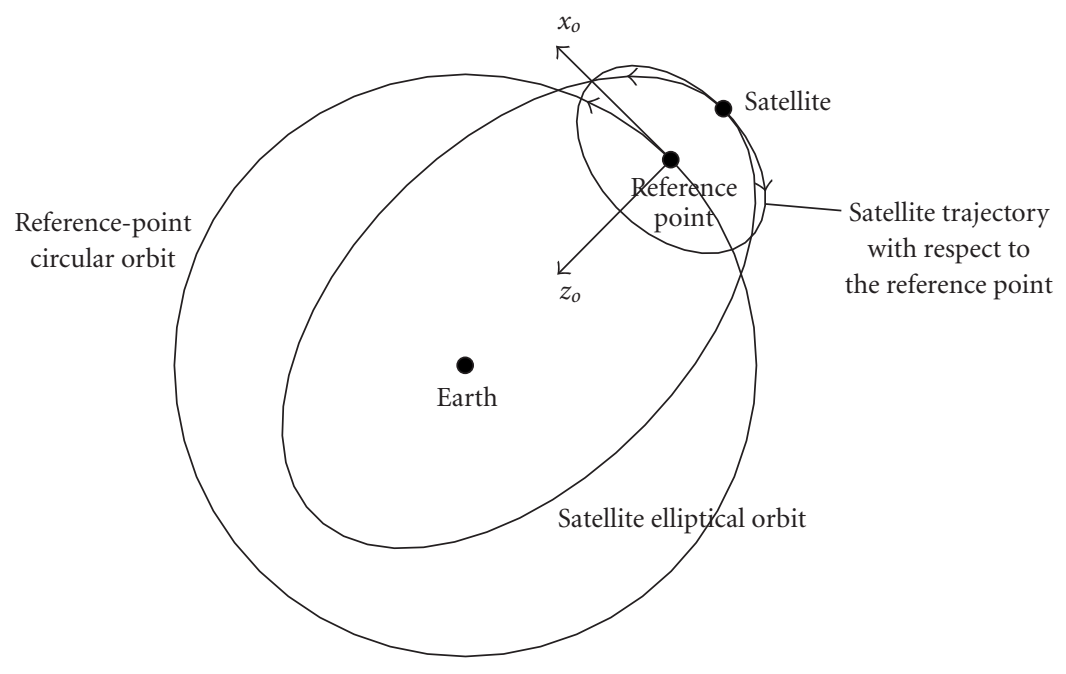

FIGURE 4: Satellite and reference point motion, plotted with satellite at apogee (not in scale for clarity).

tively. The angle in the ellipse plane varies with a constant rate (coincident with the satellite mean motion), in opposite direction with respect to the orbital motion, as shown in Figure 4 .

If the assumption (18) is discarded, the series expansion is more complicated, but similar conclusions can be obtained, with the approximated ellipse translated and rotated with respect to $x_{o} z_{o}$ axes.

Obviously, the elliptical approximation is more and more inaccurate when orbit eccentricity increases [23], which is not our case.

\subsection{Cartwheel}

The interferometric cartwheel, introduced and patented by Massonnet $[8,9]$, is basically a formation of passive microsatellites forming an orbiting cartwheel in the orbital plane of an active one, thanks to adequate differences in perigee positions and true anomalies synchronization. All satellites exhibit the same orbit eccentricity and semimajor axis. Multiple along-track and vertical baselines can be simultaneously achieved, although varying along the orbit. Obviously, increasing the number of microsatellites, formation duty cycle can be greatly improved [24]. This is not applicable to COSMO-BISSAT formation, since only two platforms are available. However, it is interesting to investigate limits and potentialities of cartwheel configuration also in this case.

First of all, considering that COSMO is in sunsynchronous low-eccentricity orbit, a Keplerian circular orbit with radius equal to COSMO semimajor axis $(6997.940 \mathrm{~km})$ has been selected as reference. Hence, COSMO and BISSAT form a cartwheel around the circular trajectory, as a consequence of their equal eccentricity (Figure 5).

From the linearized equations of motion (19), in order to obtain that the two satellites occupy the same positions in the orbiting reference frame, with a time delay $\Delta t$, we must impose

$$
\begin{aligned}
& M_{C}(0)=\theta_{0}-\omega_{C} \\
& M_{B}(0)=\theta_{0}-\omega_{B}
\end{aligned} \Longrightarrow M_{C}(0)-M_{B}(0)=\omega_{B}-\omega_{C}=\gamma=n \cdot \Delta t=f \cdot \pi
$$

Considering the trajectories in the orbiting reference frame (for the sake of simplicity, it has been assumed that at $t=$ 0 , COSMO is at its perigee), $\gamma$ is the angular separation between the satellites (Figure 6), and it can be expressed by multiplying the reference orbit mean motion times the required time separation between the satellites, or, more conveniently for constellation tuning, as a fraction $(f)$ of $\pi$.

As an example, Figure 7 reports cross-track (XTI, necessarily vertical since orbits are coplanar) and along-track (ATI) interferometric baselines, for $f=0.0833\left(\gamma=15.0^{\circ}\right.$, $\Delta t=243$ seconds). 


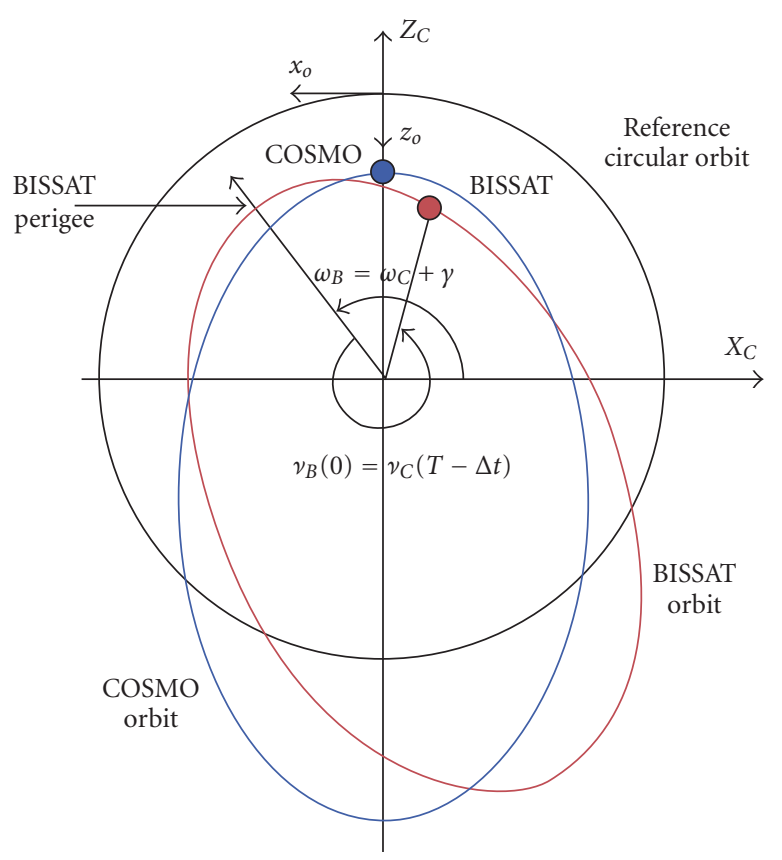

FIgURE 5: Geometry adopted to describe the COSMO-BISSAT cartwheel (plotted with COSMO at perigee and not in scale for clarity).

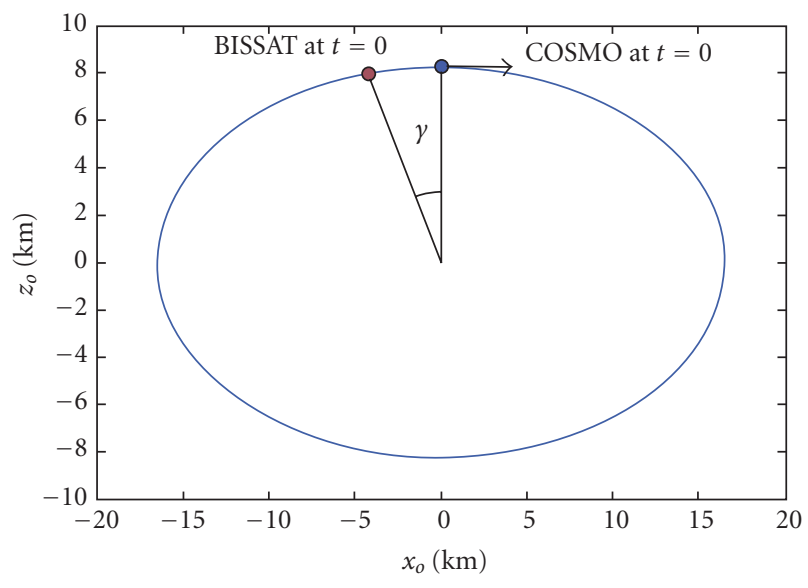

Figure 6: Motion in the $\mathrm{ORF}_{O}$.

Figure 8 and Table 2 summarize the main performances achievable with this configuration.

It is worth noting that adequate vertical baselines can be achieved almost along the whole orbit (only polar regions are excluded).

\subsection{Alternative coplanar configurations}

Two alternative solutions for baseline formation, still based on coplanar orbits, are achievable by orbiting COSMO and BISSAT with different eccentricities and equal argument of perigee and mean anomaly (Figure 9), or by choosing a

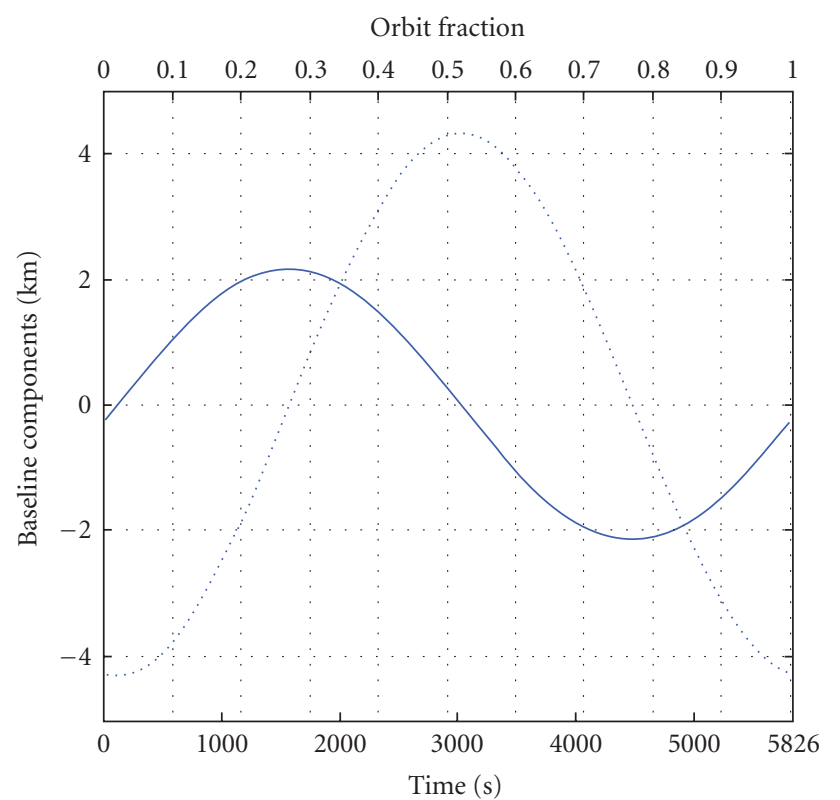

Along-track baseline

— Vertical baseline

Figure 7: Interferometric baseline components for $f=0.0833$ as a function of time along one orbit.

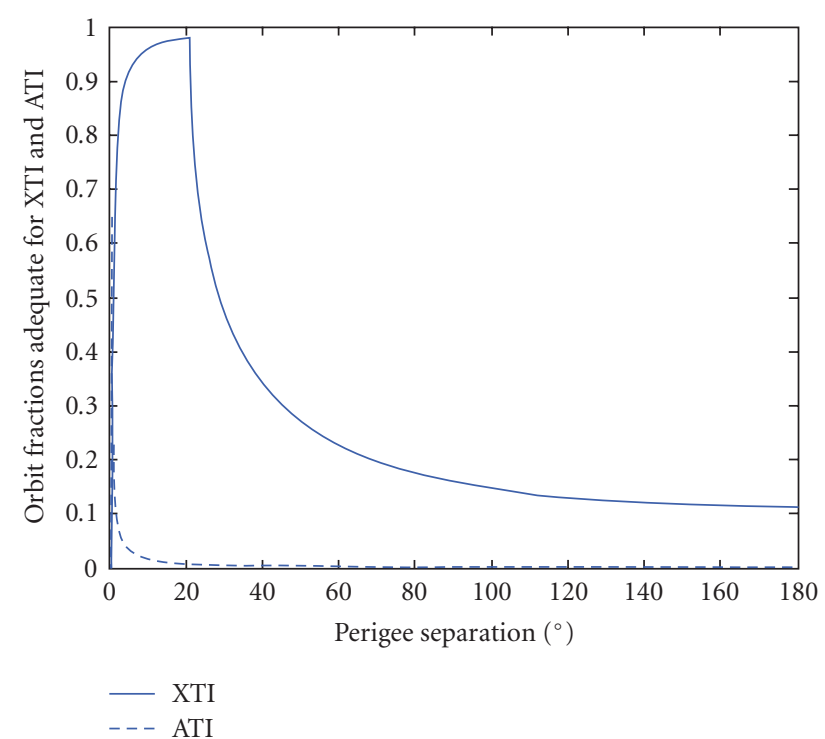

FIGURE 8: COSMO-BISSAT cartwheel performance as a function of $\gamma$, expressed in terms of fraction of orbit where satisfactory baselines are achieved.

trajectory for BISSAT which differs from the COSMO one only in the argument of perigee (Figure 10).

In these cases, the two satellites will not move on the same ellipse, with respect to the $\mathrm{ORF}_{O}$. In the first configuration, they will describe two concentric ellipses, as it is evident from (19), while in the second one the two ellipses, equal in 


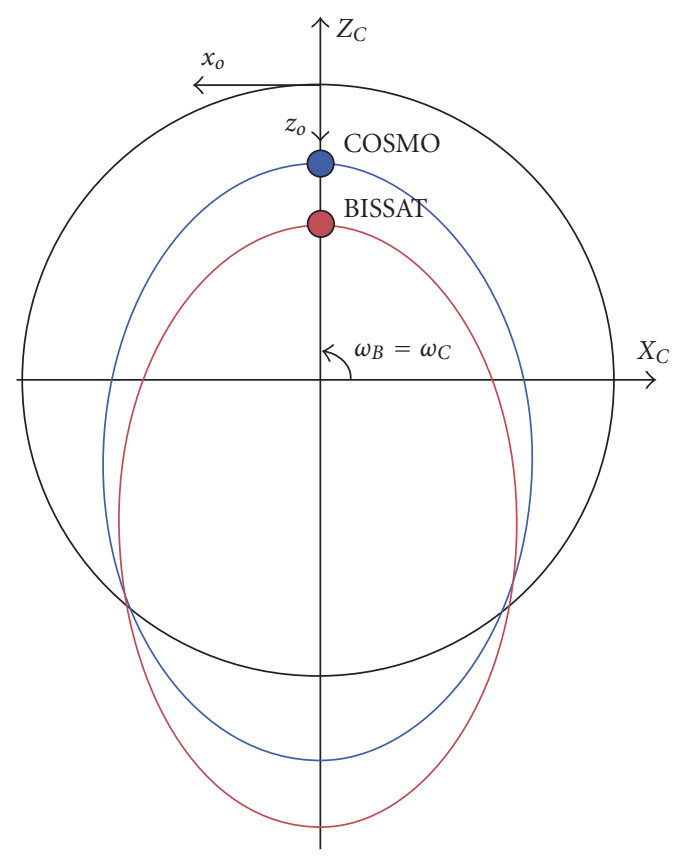

FIGURE 9: Orbits with different eccentricities (not in scale for clarity).

dimensions, will be translated on $x_{o}$-axis (this can be shown performing the series expansion in powers of eccentricity without the assumption (18), and supposing $M(0)-\theta_{0}+\omega$ small).

From the relative motion point of view, having two orbits which differ only in perigee argument is equivalent to put the two satellites on the same orbit, with a short time separation: the $\mathrm{ORF}_{O}$ coordinates of the ellipse origin are, in fact, $a \sin \left[M(0)+\omega-\theta_{0}\right]$ and $a-a \cos [M(0)+\omega-$ $\left.\theta_{0}\right]$. The along-track baseline component is constant, and to achieve an adequate vertical baseline, a large along-track separation $(>50 \mathrm{~km}$ ) is needed. As a consequence, this configuration can be used only for ATI; in particular, if $\omega_{B} \in$ $\left[89.9988^{\circ}, 89.9994^{\circ}\right]$, the entire orbit can be exploited.

In the case of different eccentricities, from the baselines' point of view, a different behavior from the cartwheel is exhibited. In fact, as it is evident from Figure 11 (still supposing that at $t=0$, COSMO is at its perigee), the vertical spacecraft separation is maximum at the poles and minimum at the ascending/descending nodes, while the along-track baseline is maximum at the nodes.

The achievable performances are quantitatively similar to the cartwheel ones, as shown in Figure 12 and Table 3, and it is interesting to point out the difference in useful latitude intervals consequent to the different trend of baseline components.

\section{TANDEM FLIGHT IN PENDULUM CONFIGURATION}

The wording "pendulum," introduced in [7, 10, 25], refers to orbits separated in the right ascension of the ascending node and, if required, with different inclinations and true anomalies (Figures 2 and 13).

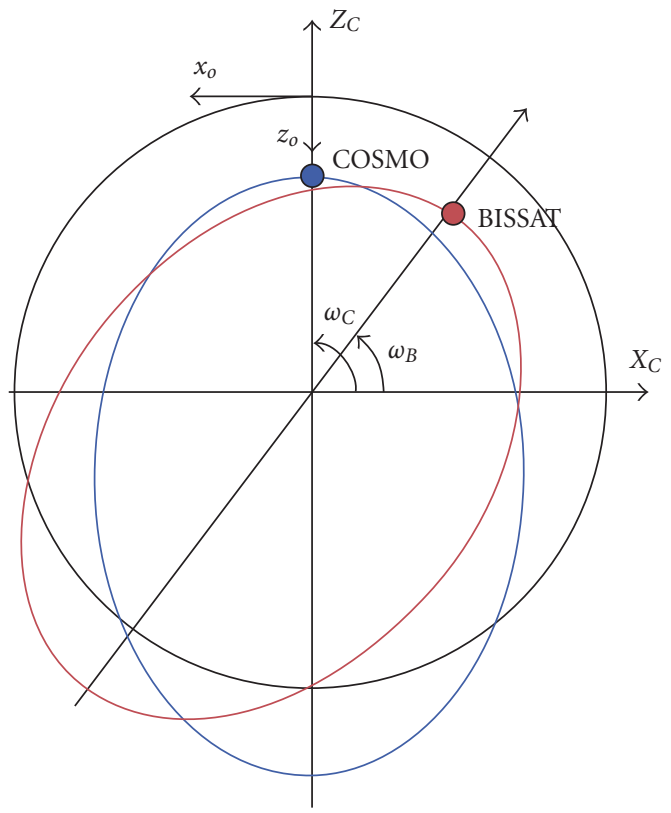

FIGURE 10: Orbits with different arguments of perigee (not in scale for clarity).

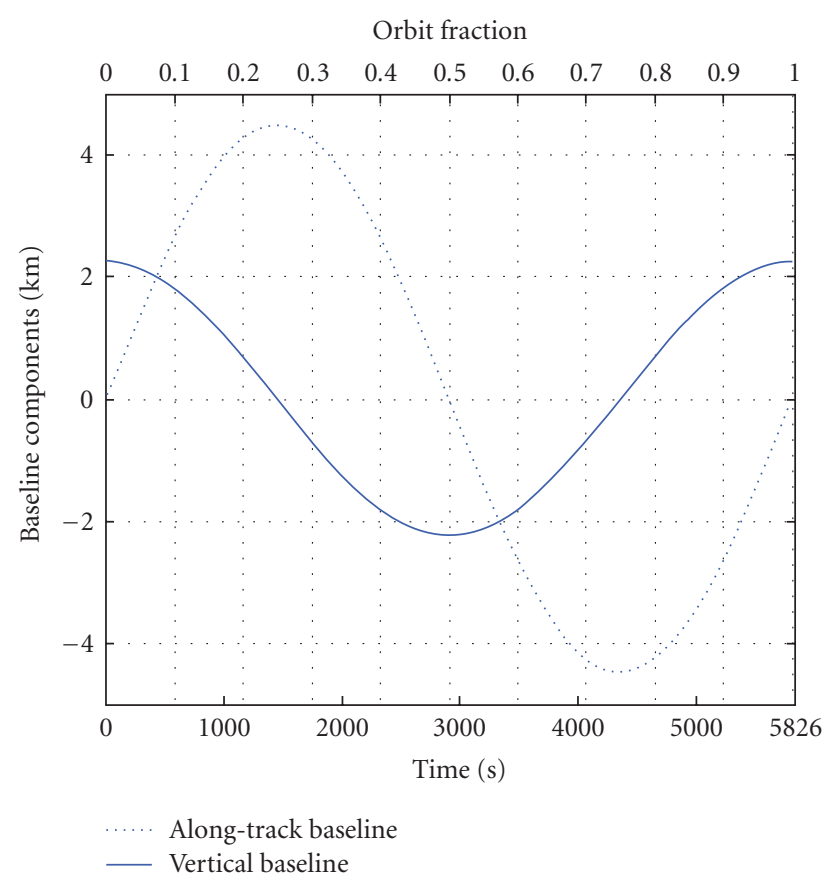

FIGURE 11: Interferometric baseline components along one orbit, for $e_{B}=1.5 \cdot 10^{-3}$.

In order to have " $J_{2}$-invariant" orbits $[26,27]$, it is appropriate to choose equal inclinations.

The pendulum relative kinematics can be better understood expanding the baseline components in a Taylor series about the points $\Delta \Omega=0, \Delta \nu=0$, and truncating beyond the first-order term in the approximation of circular orbits [21]; 
TABLE 2: Cartwheel phasings which maximize percentage of orbit adequate for XTI and ATI and consequent latitude intervals during ascending/descending phases.

\begin{tabular}{lcr}
\hline Interferometric configuration & XTI & ATI \\
\hline Perigee separation $\left(^{\circ}\right)$ & 20.74 & 0.52 \\
\hline Time separation $(\mathrm{s})$ & 335.6 & 8.41 \\
\hline Minimum distance $(\mathrm{km})$ & 2.97 & 0.0749 \\
\hline Orbit fraction $(\%)$ & 97.94 & 66.64 \\
\hline & {$[82.13,78.49]$ desc. } & {$[82.13,29.41]$ desc. } \\
Latitude intervals $\left(^{\circ}\right)$ & {$[75.56,-78.38]$ desc. } & {$[-30.02,-29.52]$ desc/asc. } \\
& {$[-75.45,82.13]$ asc. } & {$[29.91,82.13]$ asc. } \\
\hline
\end{tabular}

thus, recalling $x y z$ as the orbiting reference frame whose origin coincides with COSMO position, and assuming that at $t=0$, COSMO is at the ascending node, the following equation can be derived to express BISSAT position with respect to COSMO:

$$
\left[\begin{array}{l}
x(t) \\
y(t) \\
z(t)
\end{array}\right] \cong\left[\begin{array}{c}
a(\Delta v+\Delta \Omega \cos i) \\
a \sin i \Delta \Omega \cos (n t) \\
0
\end{array}\right]
$$

It is worth noting that cross-track baseline is formed in the horizontal plane and depends only on $\Delta \Omega$, while along-track baseline is constant and, for the considered value of sunsynchronous inclination, depends above all on $\Delta \nu$. In this case, the two spacecrafts move along almost parallel trajectories, for short orbital segments, whereas the horizontal baseline component varies as a function of latitude over longer periods [28]. In particular, from the second component of (21), the optimal value of $\Delta \Omega$ for XTI can be estimated by imposing $y_{\max }=B_{y \max }$, resulting $\Delta \Omega=0.0163^{\circ}$. The numerical simulations, performed taking into account the slight eccentricities of the orbits, confirmed this estimate, as shown in Figure 14. In order to get an adequate along-track separation, $\Delta v=-5 \cdot 10^{-3 \circ}$ has been assumed.

As for along-track interferometry is concerned, with this configuration we can achieve an ideal observation geometry. In fact, it must be considered that Earth rotation prevents two antennas, which move on the same orbit with a time separation $\Delta t$, from having the same viewing geometry.

As shown in [21], imposing the conditions $\left(\Omega_{E}\right.$ is Earth rotation rate)

$$
\frac{\Omega_{B}-\Omega_{C}}{\Omega_{E}-\dot{\Omega}}=\frac{\nu_{C}-\nu_{B}}{\dot{M}+\dot{\omega}}=\Delta t,
$$

the two antennas will exhibit the same trajectory, with respect to an Earth-fixed, rotating reference frame Figure 15. Obviously, the two satellites will have the same ground track too, thus allowing coverage geometry adequate for ATI.

Considering, for the sake of simplicity, the unperturbed case $\left(n=\sqrt{\mu / a^{3}}\right)$

$$
\frac{\Omega_{B}-\Omega_{C}}{\Omega_{E}}=\frac{\nu_{C}-\nu_{B}}{n}=\Delta t,
$$

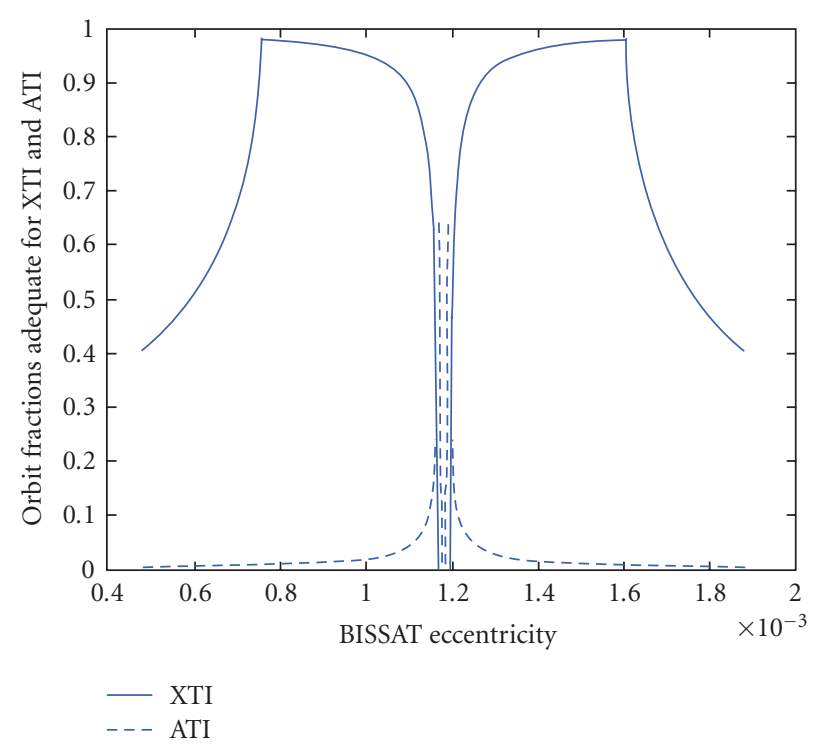

FIGURE 12: Configuration performances as a function of BISSAT eccentricity, expressed in terms of fraction of orbit where satisfactory baselines are achieved.

and choosing $\Delta t=10$ milliseconds (corresponding to an along-track baseline of about the order of $75 \mathrm{~m}$, which allows to evaluate a $V r_{\max }$ of the order of $1.56 \mathrm{~m} / \mathrm{s}$ ), the following values are derived:

$$
\begin{gathered}
\Omega_{B}-\Omega_{C}=4.18 \cdot 10^{-5 \circ} \\
\nu_{C}-\nu_{B}=6.18 \cdot 10^{-4 \circ}
\end{gathered}
$$

Relevant results are summarized in Table 4, showing the excellent ATI performance achievable with pendulum tandem configuration.

Regarding cross-track interferometry, as previously noted, in the presented configurations (cartwheel, $\Delta e$, pendulum) the differences in baseline trend lead to various useful latitude intervals. Moreover, in the common latitude intervals there is a difference in effective baseline and so in the interferometric performance (phase ambiguity and DEM accuracy). In fact, cross-track separation is larger at the equator for pendulum and cartwheel, and at the poles in the case 
TABLE 3: BISSAT eccentricities which maximize percentage of orbit adequate for XTI and ATI and consequent latitude intervals during ascending/descending phases.

\begin{tabular}{lcr}
\hline Interferometric configuration & XTI & ATI \\
\hline BISSAT eccentricity & $7.62 \cdot 10^{-4}$ & $1.169 \cdot 10^{-3}$ \\
\hline Minimum distance $(\mathrm{km})$ & 2.89 & 0.0749 \\
\hline Orbit fraction $(\%)$ & 97.92 & 66.61 \\
\hline Latitude intervals $\left({ }^{\circ}\right)$ & {$[82.13,1.94]$ desc. } & {$[59.02,-59,03]$ desc. } \\
& {$[-1.77,-1.81]$ desc/asc. } & {$[-59.06,58.99]$ asc. }
\end{tabular}

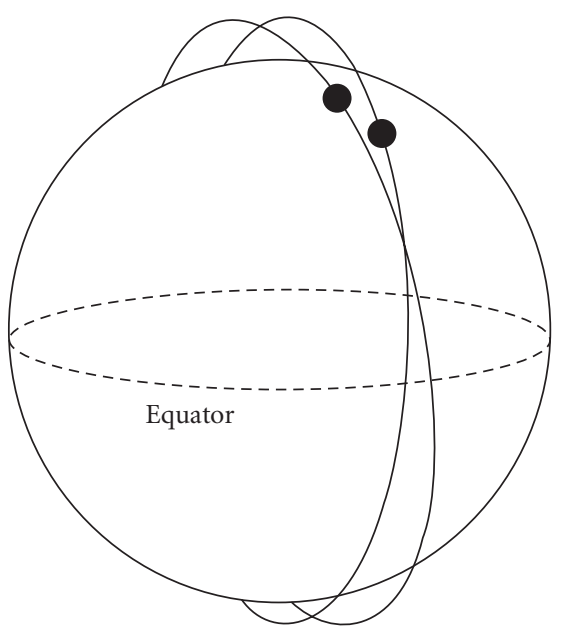

FIGURE 13: Geometry of pendulum configuration.

of different eccentricities. So, if interferometric coverage is requested at a particular latitude with a given effective baseline, proposed orbits can fulfill it either by tuning the design parameters for a certain configuration or by combining different configurations (e.g., combining cartwheel or pendulum with a difference in eccentricity). Of course, identified orbital configuration will be less effective, or useless in the worst case, at other latitudes.

On the other hand, one can think of using different orbital configurations to achieve different interferometric pairs on a given area, to combine the advantages of large and small baseline (accuracy and easier phase unwrapping), though there will be some unavoidable temporal gap between the acquisitions. To this end, optimal strategies for orbit transfer, accounting for spacecraft resources, will be addressed by further studies.

As a matter of fact, ATI can be achieved in a more stable fashion along the orbit, although with critically short baselines, whereas XTI baselines can be achieved with safer orbital configurations, although criticality arises in this case from continuous baseline variations.

\section{CONSIDERATIONS ON THE STABILITY OF COPLANAR AND PENDULUM CONFIGURATIONS}

So far, orbit fractions, where satisfactory baselines are achieved, have been evaluated assuming unperturbed motion. However, one must consider orbital perturbations to

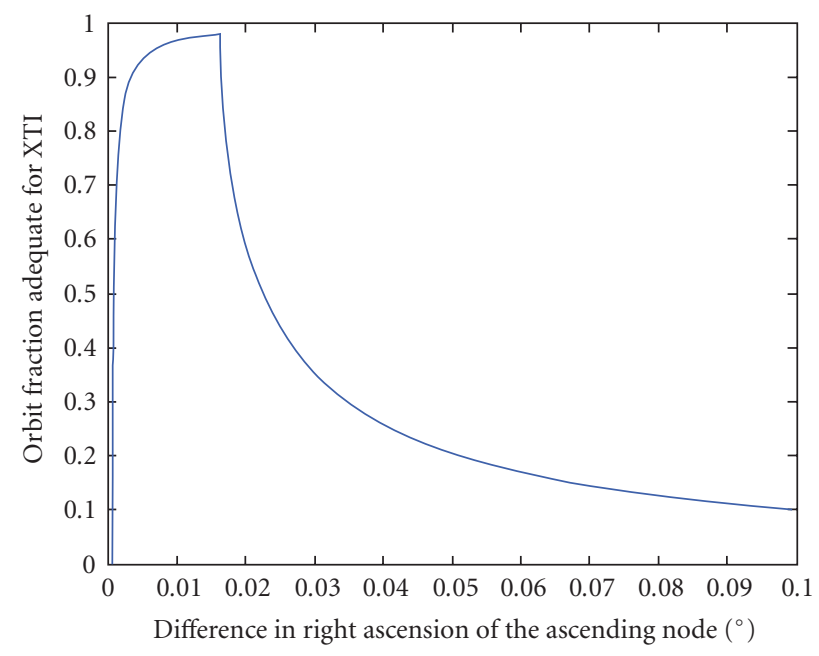

FIGURE 14: Pendulum performance as a function of $\Delta \Omega$, expressed in terms of fraction of orbit where a satisfactory XTI baseline is achieved.

estimate the long period evolution of designed configurations. To this end, it must be considered the boundary constraint deriving from the fact that we are not dealing with an original formation, on the contrary BISSAT strategies for baseline control will strongly depend on the operative schedule of the active satellite, which, as previously underlined, cannot be modified.

In this context, it is evident that, being a dual-use constellation aimed at monitoring and surveillance for commercial, scientific, and military applications, COSMO will be characterized by an accurate orbit control that counteracts the effects of drag, solar radiation pressure, and third-body accelerations. Regarding these perturbations, it can be foreseen that, for BISSAT, the firing sequence and the propellant expense should be similar to COSMO ones. It is expected that this is particularly true if the same bus of COSMO will be adopted for BISSAT.

In more detail, in the case of orbits with different eccentricities, the differential drag is more consistent, while, for all the other configurations, the aerodynamic effect, integrated over one orbital period, is the same for the two satellites, assuming that they are almost identical and neglecting atmospheric randomness, attitude dynamics and differences in fuel consumption. 
TABLE 4: Pendulum configurations which maximize percentage of orbit adequate for XTI and ATI and consequent latitude intervals during ascending/descending phases.

\begin{tabular}{lcc}
\hline Interferometric configuration & XTI & ATI \\
\hline$\Delta \Omega\left(^{\circ}\right)$ & 0.0162 & $4.18 \cdot 10^{-5 \circ}$ \\
\hline$\Delta \nu\left(^{\circ}\right)$ & $-5 \cdot 10^{-3}$ & $-6.18 \cdot 10^{-4 \circ}$ \\
\hline Minimum distance $(\mathrm{km})$ & 0.879 & 0.075 \\
\hline Orbit fraction $(\%)$ & 97.93 & 100 \\
\hline Latitude intervals $\left(^{\circ}\right)$ & {$[81.92,-81,91]$ desc. } & All the achievable latitudes \\
\hline
\end{tabular}

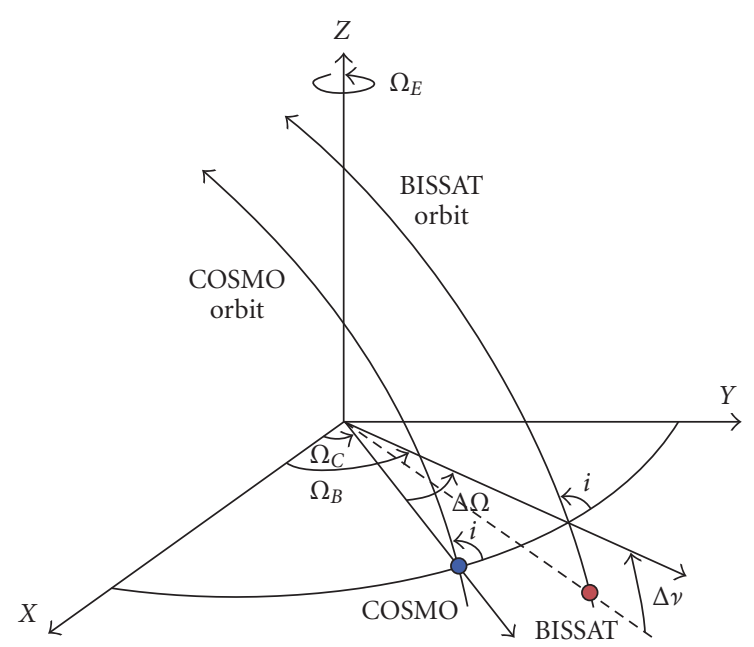

FIGURE 15: Along-track interferometer: positions at the ascending node.

As for nonspherical Earth effects are concerned, COSMO argument of perigee will be kept in a certain range around $90^{\circ}$, by nullifying the $J_{2}$ induced precession of the line of apsides. This effect is moderate since COSMO orbit is frozen. From formation keeping point of view, the configuration with different eccentricities is the only one that is not $J_{2}$ invariant. However, differential secular $J_{2}$ effects on the evolution of the interferometric baseline are negligible in the considered case, as it can be seen in Figures 16 and 17, where relative trajectory is reported for $e_{B}=0.00140$, and for 450 COSMO nodal periods. In this simulation, only $J_{2}$ secular effects have been considered (without any correction): it can be seen that the growth of baseline horizontal component is so slow that, after one month, the secular value is still smaller than $5 \mathrm{~m}$.

This is due to the fact that, for near circular orbits, $\dot{\Omega}$, $\dot{\omega}$, and $\dot{M}$ are much more sensitive to $\Delta i$ than to $\Delta e$ (in fact, $\partial \dot{\Omega} /\left.\partial e\right|_{e=0}=\partial \dot{M} /\left.\partial e\right|_{e=0}=\partial \dot{\omega} /\left.\partial e\right|_{e=0}=0$ ), so the differences in mean anomaly, argument of perigee, and right ascension of the ascending node are, in the considered case, of order $10^{-30} / y$.

As for latitude coverage is concerned, it is obvious that if the precession of the line of apsides were not counteracted, the latitude intervals in which interferometry is possible, with certain baselines, would be altered. As previously stated, argument of perigee control is envisaged in COSMO operative schedule because of strict repetitiveness requirements. As for BISSAT, only in the pendulum case, passive satellite orbit is frozen; in the cartwheel case, for example, argument of perigee control will be more onerous, leading to a (presumibly slight) difference in fuel consumption.

To summarize, pendulum is the stablest configuration, followed by cartwheel (not frozen) and $\Delta e$ (not $J_{2}$-invariant).

\section{CONCLUSIONS}

This paper focused on orbital configurations adequate for complementing the Italian COSMO SAR constellation with interferometry. A fifth satellite has been considered that, thanks to expectable mass reductions consequent to a simplified passive payload, could offer additional maneuvers capabilities, thus allowing, as an example, mission changes from along-track to cross-track interferometry, depending on particular users' requirements. To this end, further studies will be addressed to characterize optimal strategies for orbit transfer. Enlarged maneuvers capabilities could also allow flight of the fifth satellite in formation with a varying COSMO spacecraft, thus achieving an overall reliability improvement. In addition, it is worth noting that the proposed idea of a passive satellite can be fulfilled by considering only recurrent costs or by using most of the engineering model of the spacecraft. On the other hand, the weight, volume, and cost advantages connected with the use of a simplified, receiving-only payload could allow additional remote sensing systems to be embarked, such as a laser altimeter or an atmospheric profiler which could take advantage of the COSMO terminator orbit too.

The paper described a general purpose model developed for propagating tandem configurations and for evaluating relevant geometric performance. The model was applied considering as input the most referenced orbital configurations for interferometric applications and accounting for major limiting factors in baseline time hystories along the orbit. Numerical simulation results pointed out that the most favorable tandem configuration for along-track interferometry (allowing continuous coverage with constant along-track baseline) consists in pendulum tandem configuration, with 


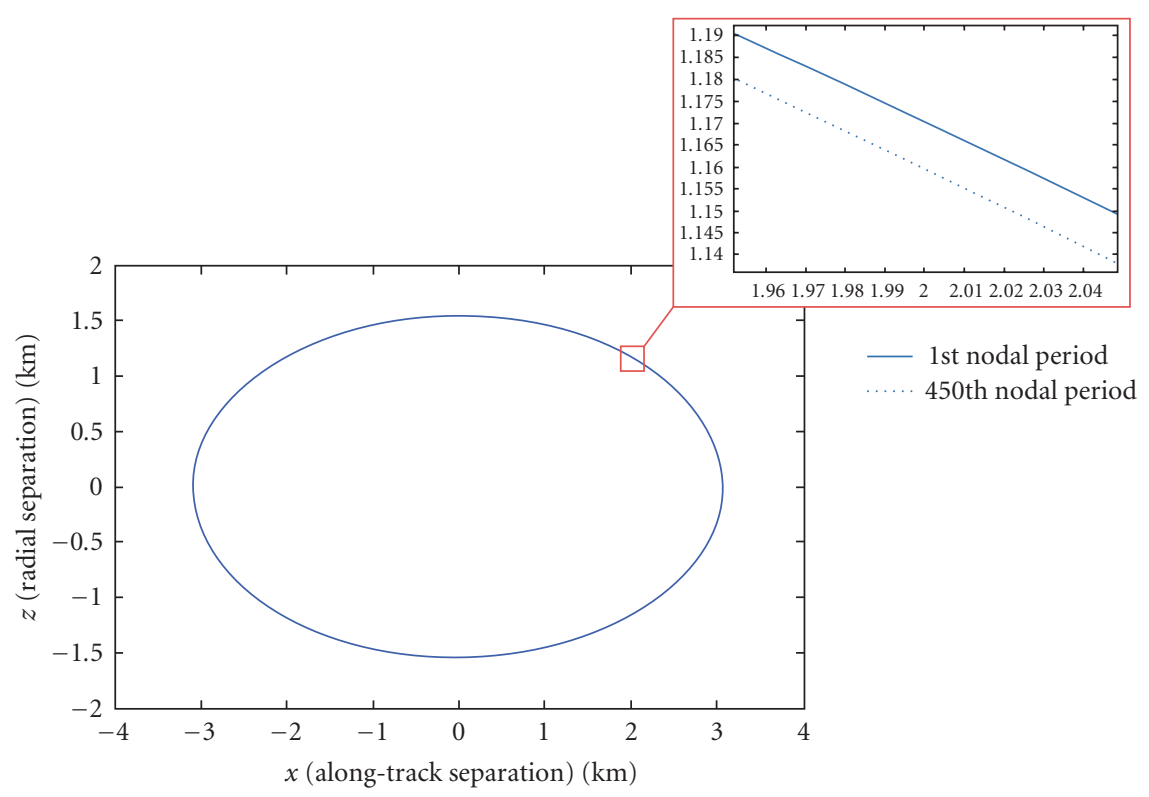

FIGURE 16: In-plane relative trajectory, plotted for $e_{B}=0.00140$ and 450 nodal periods.

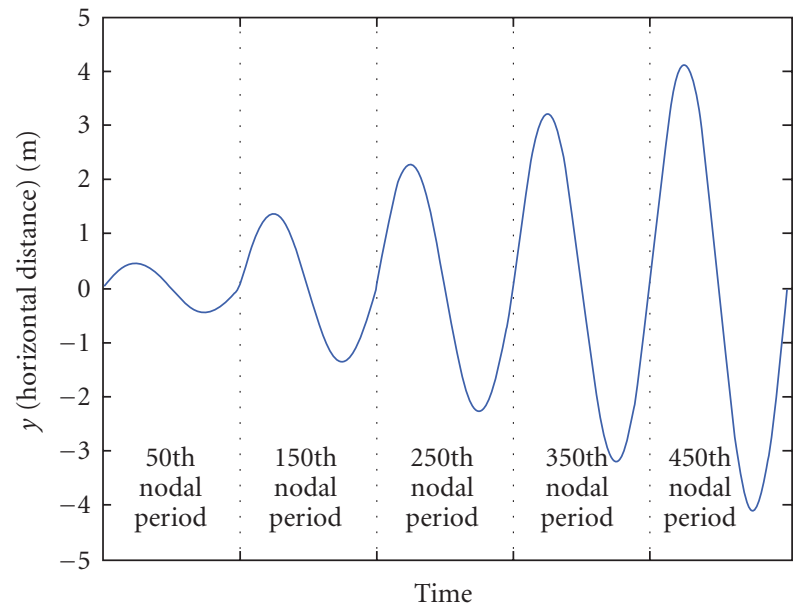

FIGURE 17: Secular growth of baseline horizontal component, plotted for $e_{B}=0.00140$.

ascending node and true anomaly separations adequate to match Earth rotation. Regarding cross-track interferometry, developed model allowed identification of several solutions which enable coverage for more than $90 \%$ of the orbit. Furthermore, it was shown that by tuning orbital parameters such as perigee, ascending node, anomaly separation, or orbit eccentricity, it is possible to set the latitude interval in which cross-track SAR interferometry is carried out with selected horizontal or vertical baseline.

\section{ACKNOWLEDGMENT}

This paper has been carried out with financial support from the Italian Space Agency and Ministry for Education, University and Research.

\section{REFERENCES}

[1] F. Caltagirone, G. Angino, A. Coletta, F. Impagnatiello, and A. Gallon, "COSMO-SkyMed program: Status and perspectives," in Proc. 3rd International Workshop on Satellite Constellations and Formation Flying, pp. 11-16, Pisa, Italy, February 2003.

[2] F. Caltagirone, P. Spera, G. Manoni, and L. Bianchi, "COSMO-SkyMED: A dual use earth observation constellation," in Proc. 2nd International Workshop on Satellite Constellations and Formation Flying, pp. 87-94, Haifa, Israel, February 2001.

[3] A. Moccia, N. Chiacchio, and A. Capone, "Spaceborne bistatic synthetic aperture radar for remote sensing applications," International Journal of Remote Sensing, vol. 21, no. 18, pp. 3395-3414, 2000.

[4] M. D'Errico and A. Moccia, "Attitude and antenna pointing design of bistatic radar formations," IEEE Trans. Aerosp. Electron. Syst., vol. 39, no. 3, pp. 949-960, 2003.

[5] H. A. Zebker and J. Villasenor, "Decorrelation in interferometric radar echoes," IEEE Trans. Geosci. Remote Sensing, vol. 30, no. 5, pp. 950-959, 1992.

[6] G. Krieger, H. Fiedler, J. Mittermayer, K. Papathanassiou, and A. Moreira, "Analysis of multistatic configurations for spaceborne SAR interferometry," IEE Proceedings - Radar, Sonar and Navigation, vol. 150, no. 3, pp. 87-96, 2003.

[7] G. Krieger, M. Wendler, H. Fiedler, J. Mittermayer, and A. Moreira, "Performance analysis for bistatic interferometric SAR configurations," in Proc. IEEE International Geoscience and Remote Sensing Symposium (IGARSS '02), vol. 1, pp. 650652, Toronto, Canada, June 2002.

[8] D. Massonnet, "Capabilities and limitations of the interferometric cartwheel," IEEE Trans. Geosci. Remote Sensing, vol. 39, no. 3, pp. 506-520, 2001.

[9] D. Massonnet, "The interferometric cartwheel: a constellation of passive satellites to produce radar images to be coherently combined," International Journal of Remote Sensing, vol. 22, no. 12, pp. 2413-2430, 2001.

[10] H. Fiedler, G. Krieger, F. Jochim, M. Kirschner, and A. Moreira, "Analysis of bistatic configurations for spaceborne SAR interferometry," in Proc. 4th European Conference on Synthetic 
Aperture Radar (EUSAR '02), pp. 29-32, Cologne, Germany, June 2002.

[11] H. A. Zebker, T. G. Farr, R. P. Salazar, and T. H. Dixon, "Mapping the world's topography using radar interferometry: the TOPSAT mission," Proc. IEEE, vol. 82, no. 12, pp. 1774-1786, 1994.

[12] M. D'Errico, A. Moccia, and S. Vetrella, "High frequency observation by GTM antenna range beam steering," EARSeL Advances in Remote Sensing, vol. 3, no. 1-IX, pp. 60-69, 1994.

[13] R. Gens and J. L. van Genderen, "SAR interferometryissues, techniques, applications," International Journal of Remote Sensing, vol. 17, no. 10, pp. 1803-1836, 1996.

[14] P. A. Rosen, S. Hensley, I. R. Joughin, et al., "Synthetic aperture radar interferometry," Proc. IEEE, vol. 88, no. 3, pp. 333$382,2000$.

[15] A. Moccia, S. Esposito, and M. D’Errico, "Height measurement accuracy of ERS-1 SAR interferometry," EARSeL Advances in Remote Sensing, vol. 3, no. 1, pp. 94-108, 1994.

[16] F. K. Li and R. M. Goldstein, "Studies of multibaseline spaceborne interferometric synthetic aperture radars," IEEE Trans. Geosci. Remote Sensing, vol. 28, no. 1, pp. 88-97, 1990.

[17] E. Rodriguez and J. M. Martin, "Theory and design of interferometric synthetic aperture radars," IEE Proceedings F, Radar \& Signal Processing, vol. 139, no. 2, pp. 147-159, 1992.

[18] A. Moccia and S. Vetrella, "A tethered interferometric synthetic aperture radar (SAR) for atopographic mission," IEEE Trans. Geosci. Remote Sensing, vol. 30, no. 1, pp. 103-109, 1992.

[19] R. Bamler and P. Hartl, "Synthetic aperture radar interferometry," Inverse Problems, vol. 14, no. 4, pp. R1-R54, 1998.

[20] R. Romeiser, M. Schwäbisch, J. Schulz-Stellenfleth, et al., "Study on concepts for radar interferometry from satellites for ocean (and land) applications (KoRIOLiS)," Final Report, University of Hamburg, Hamburg, Germany, 2002.

[21] A. Moccia and G. Rufino, "Spaceborne along-track SAR interferometry: performance analysis and mission scenarios," IEEE Trans. Aerosp. Electron. Syst., vol. 37, no. 1, pp. 199-213, 2001.

[22] V. A. Chobotov, Ed., Orbital Mechanics, AIAA Education Series, American Institute of Aeronautics and Astronautics, Washington, DC, USA, 1991.

[23] A. Miele and M. D’Errico, “A relative orbital motion model oriented to formation design," in Proc. 3rd International Workshop on Satellite Constellations and Formation Flying, Pisa, Italy, February 2003.

[24] D. Massonnet, E. Thouvenot, S. Ramongassiè, and L. Phalippou, "A wheel of passive radar microsats for upgrading existing SAR projects," in Proc. IEEE International Geoscience and Remote Sensing Symposium (IGARSS '00), vol. 3, pp. 10001003, Honolulu, Hawaii, USA, July 2000.

[25] J. Mittermayer, G. Krieger, A. Moreira, and M. Wendler, "Interferometric performance estimation of the interferometric cartwheel in combination with a transmitting SAR-satellite," in Proc. IEEE International Geoscience and Remote Sensing Symposium (IGARSS '01), vol. 7, pp. 2955-2957, Sydney, NSW, Australia, July 2001.

[26] H. Schaub, S. R. Vadali, J. L. Junkins, and K. T. Alfriend, "Spacecraft formation flying control using mean orbit elements," in Proc. AAS/AIAA Astrodynamics Specialists Conference, Girdwood, Alaska, August 1999, Paper No. AAS 99-310.

[27] H. Schaub and K. T. Alfriend, "J2 invariant relative orbits for spacecraft formations," Celestial Mechanics and Dynamical Astronomy, vol. 79, no. 2, pp. 77-95, 2001.

[28] A. Moccia, S. Vetrella, and M. D'Errico, “Twin satellite orbital and doppler parameters for global topographic mapping," EARSeL Advances in Remote Sensing, vol. 4, no. 2-X, pp. 55-66, 1995.
A. Moccia has been a Professor of aerospace servosystems at the Faculty of Engineering, University of Naples, Naples, Italy, since 1990. His research activities deal with aerospace high-resolution remote-sensing systems, mission analysis, design, and data processing, as well as aerospace systems dynamics and control. He has been a member of national and international committees and working groups (ASI, NASA, ESA, UE, CIRA, EARSA eL, MIUR) and principal or coinvestigator of several national and international research programs. He is the author or coauthor of more than 100 scientific papers, and gained several references in international journals. Since 1975, he has held several grants and contracts from the National Research Council, University of Naples, Italian Space Agency, ESA, Italian Ministry for Education, University and Research, Alenia Spazio, Telespazio, and Technapoli. Since 2001, he has been the Chairman of the Ph.D. School in Aerospace Engineering at the University of Naples and President of CORISTA, Consortium for Research on Aerospace Remote Sensing Systems, a nonprofit research consortium among Universities of Naples and Bari, Alenia Spazio, and Laben.

G. Fasano received the M.S. degree in aerospace engineering in 2004 and is currently a Ph.D. student, in aerospace engineering at the university of Naples "Federico II." His current research activities deal with interferometric and bistatic missions based on SAR constellations, formation flying dynamics, and automatic UAV senseand-avoid systems.

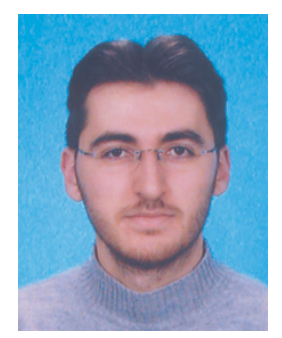

